Paper:

\title{
Analysis and Characterization of Machined Surfaces with Aesthetic Functionality
}

\author{
Francesco Giuseppe Biondani* ${ }^{* \dagger}$, Giuliano Bissacco*, Lukáš Pilný ${ }^{* *}$, and Hans Nørgaard Hansen* \\ ${ }^{*}$ Department of Mechanical Engineering, Technical University of Denmark \\ Produktionstorvet, Kgs. Lyngby 2800, Denmark \\ †Corresponding author, E-mail: frgbio@mek.dtu.dk \\ ** Novo Nordisk A/S, Hillerød, Denmark \\ [Received July 3, 2018; accepted December 5, 2018]
}

The generation of fine machined surfaces with high gloss is an important topic in mould manufacturing. The surface gloss can be characterized by means of scattered light sensors and a representative parameter such as $A q$. In this paper, in-line measurements of scattered light distribution are compared with roughness parameters calculated using a confocal microscope, in order to assess surface aesthetic quality. Several surfaces have been machined by means of high precision milling, producing different surface topographies. Surface characterization has been performed on a machine using a scattered light sensor, and using a confocal microscope in laboratory conditions. The calculated $A q$ parameter is compared with the amplitude roughness parameters $S a$ and $S q$, and with hybrid parameters $S d q$ and $R d q$ representing the average slope of the surface features. Scanning electron microscope (SEM) images are used as visual benchmarks to identify the parameters' correlation with the visual appearance. A different linear trend of the relationship between $A q, R d q$, and $S d q$ is observed. The description of the surface quality through $S a$ or $S q$ instead is found to be insufficient. This is explained by means of SEM pictures showing a dramatic influence of the smeared material over the machined surface.

Keywords: scattered light sensor, surface appearance, machining

\section{Introduction}

Within the trend of "Industry 4.0" [1], more and more quality control operations are shifting from off-line to inline applications. On a machine, data collection significantly decreases the response time in which information is made available, allowing the implementation of realtime strategies for optimizing the process itself [2], and increasing the overall efficiency and precision of the process [3].

In-line and on-the-machine monitoring of manufacturing processes becomes particularly important in production of small batches of high-value products, where on- line adjustment of a process with a short response time is required [4].

Ball end milling is a well-established machining process used for the production of free-form surfaces, in particular in the mould industry. Due to the spherical shape of the cutter, the tool can follow the curvature of the part being machined, and is therefore particularly suited for complex free-form geometries. The complex kinematic of the cutting process in ball end milling influences the topology and integrity of the generated surface [5]. When the surface has aesthetic functionality, the micro geometry, i.e., the roughness, becomes of crucial importance.

Nanometer-scale surface texture and micrometer-scale waviness are known to improve the aesthetic quality of machined parts $[2,6]$.

Surface roughness is conventionally indicated on technical drawings in terms of $R a$ and $R q$ ( $S a$ and $S q$ ). These parameters are the most common and well-known, but they provide a limited amount of information about the aesthetics of the surface. The relationship between the surface generating process, surface topography characterization, and surface functionality has been addressed in $[7,8]$.

Measurements of roughness parameters require the use of profilometers, interferometers, or optical microscopes. Thus, they are carried out off-line, because they are not suited for shop-floor measurements with uncontrolled environmental conditions [9]. Issues related to the traceability of instruments for metrology at microscale and nanoscale are discussed in [10].

Gloss is defined as "the attribute of the surfaces that causes them to have shiny or lustrous metallic appearance" [11]. It is associated with the amount of light reflected in a mirror-like direction [12], and is inherently related with the orientation of the microscopic facets composing the surface [11]. A quantitative evaluation of the average micro facets orientation can be performed through light-scattering measurement [13].

Using scattered light sensors in an industrial contest has several advantages such as: high measurement rate, fast coverage of large areas, and low sensitivity to the environmental conditions [14]. Scattered light sensors are widely used to characterize the surface texture in the semiconductor and optical industries $[9,15]$. In-process charac- 
terization of rotational symmetric polished surfaces using scattered light sensors has been demonstrated in [16].

The scope of this work is to verify the applicability of a scattered light sensor for machine characterization of ball end milled surfaces with aesthetic functionality, to be used in injection moulding tools. For this purpose, different surfaces have been machined using ball end mills. The gloss of the machined areas has been evaluated using a commercial scatterometer integrated in a five-axis milling machine in order to perform in-line (onthe-machine) measurements. The results have been compared with laboratory confocal measurements and SEM images to assess the feasibility of in-line measurements for aesthetic evaluation.

\section{Characterization of Aesthetic Surfaces}

The aesthetic requirements of mould surfaces are related to the desired surface appearance of moulded parts. In polymer injection moulding, the polymer replicates the mould surface, and therefore it is necessary to correctly evaluate the mould appearance. The quality of highgloss surfaces can be assessed by means of visual inspection. Trained operators examine the mould surfaces and decide whether the specific aesthetic requirements have been met. As this process is based on an operator's observation, it lacks repeatability and objectivity. Unbiased parameters such as $S a$ and $S q$ are roughness amplitude parameters that describe the surface in terms of deviation from the mean line of the profile. More specifically, $\mathrm{Sa}$ is the arithmetic average of the profile amplitude, while $S q$ is the average root mean square (RMS) height. Being amplitude parameters, they do not describe spatial periodicity and profile slopes, which strongly affect the visual appearance of the surface.

Direct estimation of surface gloss can be performed by means of a scattered light sensor.

The scatterometer used in this work projects a light beam toward the surface, and a diode array measures the intensity and angular distribution of the light coming back. An ideal mirror-like surface scatters the light in a specular direction. In contrast, a real surface scatters the light back with a certain angular distribution.

The variance of the scattered light angular distribution can be used to characterize surface gloss ( $A q$ parameter) [17]. The higher $A q$, the broader the intensity distribution, leading to a lower glossiness of the surface $[18,19]$.

In order to validate the applicability of scattered light measurements for evaluating surface appearance, $A q$ values are compared with SEM images of the surfaces and with two other roughness parameters, such as $S d q$ and $R d q$.

These two parameters represent the RMS of the slopes of the profile, and they are calculated through optical profilometer measurements.
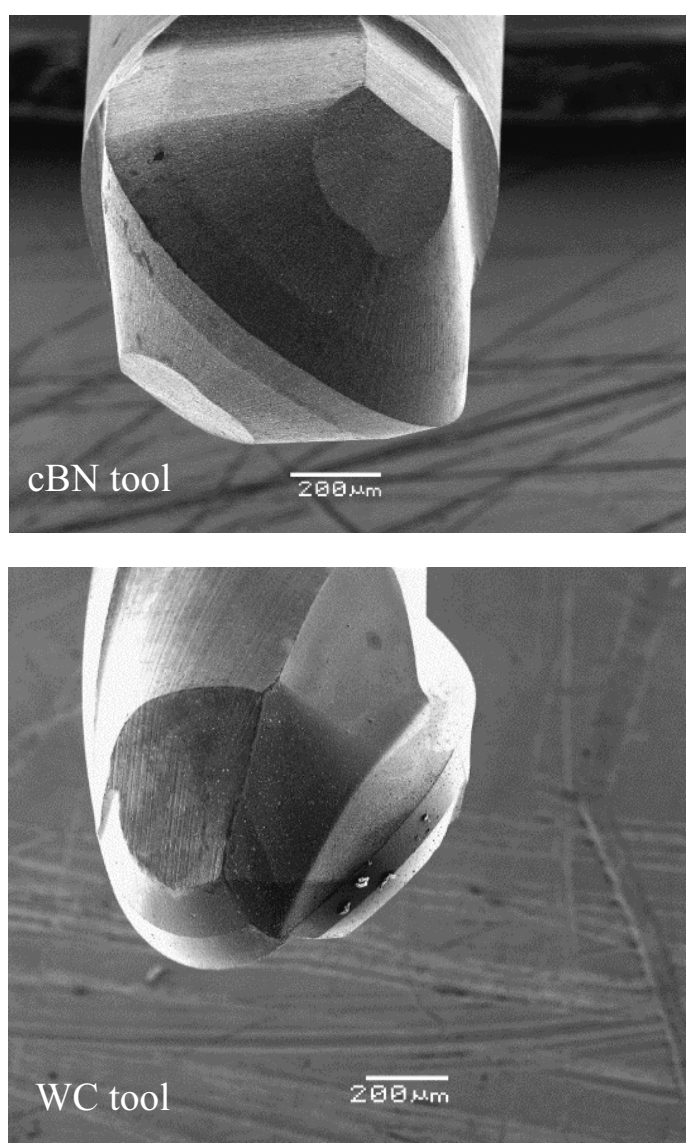

Fig. 1. On top: cubic born nitride $(\mathrm{cBN})$ tool used in the experiments. On the bottom: tungsten carbide tool (WC). The diameter for both tools is $1 \mathrm{~mm}$.

\section{Experimental Plan}

\subsection{Machining Strategy}

The specific tool geometry influences the surface appearance and therefore, two different tools have been used: a cubic boron nitride ball end mill (cBN), and a tungsten carbide ball end mill (WC), as shown in Fig. 1. Both tools were two-fluted with a radius of $0.5 \mathrm{~mm}$, but had different teeth geometries. The tools have been mounted in a Mikron HSM 400 ULP five-axis milling machine. A square tool path has been machined on a flat steel surface with an inclination angle of $40^{\circ}$ between the surface normal and the spindle axis. The particular choice of the tool path divides the test parts into four different zones that will be referred to as A, B, C, and D (Fig. 2).

Considering the tool moving clockwise, it will start machining the surface while moving vertically upward (zone A). After that, it will move horizontally downward (zone B), and again vertically downward (zone C).

Eventually, the tool will move horizontally again (zone D), and it will reach the starting point (zone A) in Fig. 2.

In each of these zones, the engagement conditions between the tool and the workpiece are different, and in turn, the topology of the generated surfaces is different. In this 


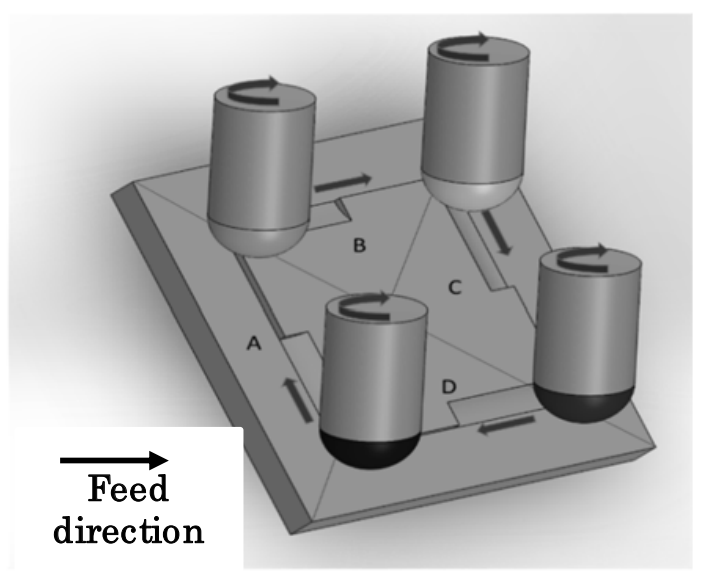

Fig. 2. Machining strategy.

way, it is possible to simulate the different cutting conditions that may occur when machining a free-form surface.

All the four zones were machined using $\mathrm{cBN}$ and a WC tool was replicated twice.

\subsection{Scattered Light Sensor Measurement Strategy}

A commercial scatterometer, OptoSurf OS 500-32, has been integrated in the five-axis milling machine, and has been used for the gloss measurements. The instrument consists of a photodiode that generates a light beam $(\lambda \sim$ $670 \mathrm{~nm}$ [20]). The beam is focused on the surface within a spot size of $0.9 \mathrm{~mm}$, and a photodiode array detects the intensity and angular position of the light scattered back from the surface. The maximum detectable scatter angle is related to the diode array length, and is approximately $\pm 16^{\circ}$, with numerical aperture $(\mathrm{NA})=0.28$. The instrument has been fixed to the machine tool spindle (the same used for surface machining), and the tool path has been programmed to scan the test part surface. Due to the high measuring rate of the Optosurf, it has been possible to scan the whole $20 \times 20 \mathrm{~mm}$ workpiece surface in $16 \mathrm{~min}$, using a scanning speed of $500 \mathrm{~mm} / \mathrm{s}$ and a hatch space of $0.1 \mathrm{~mm}$. The measurements have been repeated by scanning the workpiece parallelly and orthogonally to the feed direction of the cutting tool. The intensity of the scattered light and the $A q$ parameter have been logged on an external computer and processed with MATLAB. Five points for each surface in the in-feed and cross-feed measurement directions have been extracted from the data file with the respective value of $A q$. Care has been taken in extracting the same points on the scattered light distribution plot as those measured with the confocal instrument.

\subsection{Confocal Microscope Measurement Strategy}

The surfaces have been measured in laboratory conditions with a confocal microscope LEXT OLS 4100, using an objective lens with 50x magnification and a NA of 0.95 . The micrscope uses a blue laser in the scanning mode with a spot size of $0.2 \mu \mathrm{m}$.

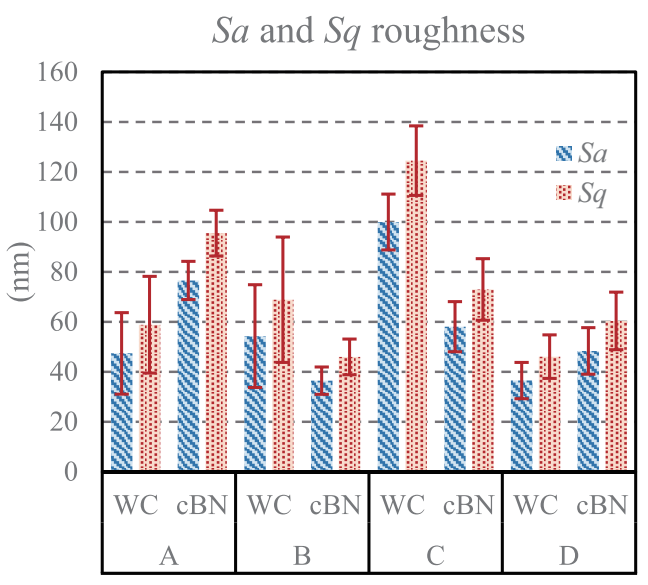

Fig. 3. $S a$ and $S q$ calculated from the confocal measurements.

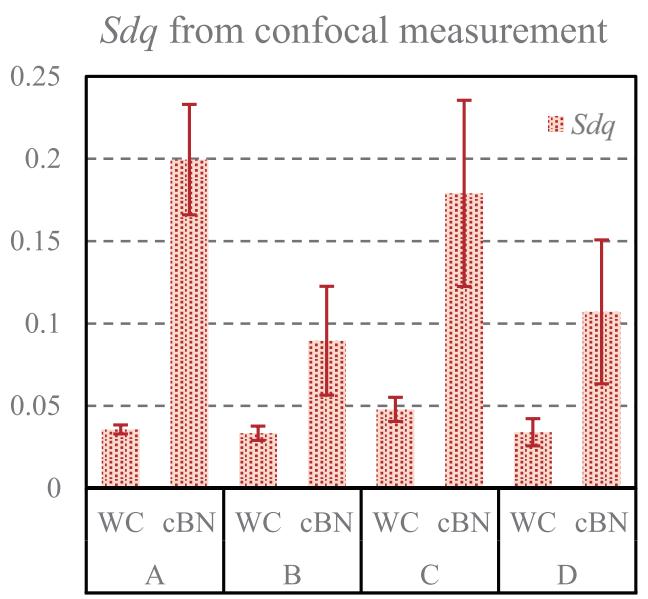

Fig. 4. $S d q$ calculated from the confocal measurements.

Five points, $250 \mu \mathrm{m} \times 250 \mu \mathrm{m}$, have been selected on the surfaces A, B, C, and D, both for the surfaces generated by the WC tool, and for those generated by the $\mathrm{cBN}$ tool. Every measurement has been repeated three times.

\section{Experimental Results}

The confocal images have been processed using SPIP Image Metrology ${ }^{\mathrm{TM}}$ software, a first-order global levelling correction has been applied to the pictures, and $S a$, $S q$, and $S d q$ have been calculated. Subsequently, five profiles along the feed direction and orthogonal to it have been extracted, and the $R d q$ parameter has been calculated and averaged for each direction. A characterization of the surfaces is shown in Fig. 3, where every column consists of the average of ten points ( 5 points per two repetitions).

The most favorable conditions in terms of $S a$ and $S q$ were achieved in zone D for the $\mathrm{WC}$ tool and in zone B for the $\mathrm{cBN}$ tool, with a minimum $S a$ roughness of $36 \mathrm{~nm}$. The other areas present higher roughness, up to $100 \mathrm{~nm}$.

The parameter $S d q$ is shown in Fig. 4. It is nearly constant in the area machined using the WC tool and dramat- 


\section{$R d q$ from confocal measurement}

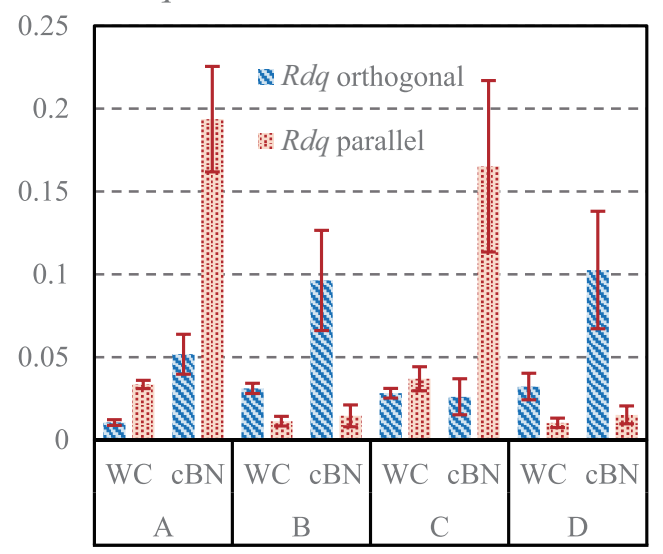

Fig. 5. $R d q$ calculated from the confocal measurements in the orthogonal and parallel direction to the tool feed direction.

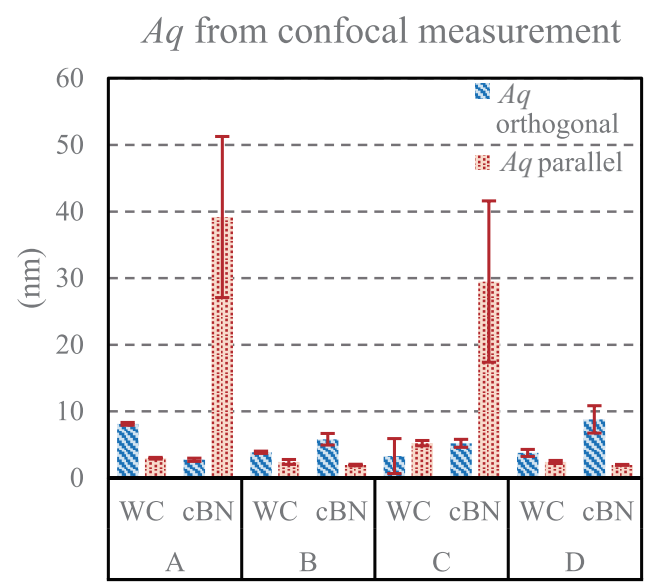

Fig. 6. $A q$ calculated from the scattered light measurements in the orthogonal and parallel direction to the tool feed direction.

ically increases when the $\mathrm{cBN}$ areas are analyzed, with areas $\mathrm{A}$ and $\mathrm{C}$ showing values much higher than areas $\mathrm{B}$ and D. The $R d q$ value is shown in Fig. 5, in both the orthogonal and parallel direction to the tool feed. It is interesting to notice that the higher values of $R d q$ are not always measured across the feed direction as expected.

In Fig. 6, the $A q$ values are presented in the orthogonal and parallel direction as well. At first glance, as expected, the trend in $A q$ values shows much more similarity to $S d q$ and $R d q$ than to the amplitude parameters $S a$ and $S q$. However, some discrepancies can be noticed in the relation between $A q, R d q$, and $S d q$; the relative differences of the values between different areas are not the same for the three parameters. To gain a better understanding of the surface topology, SEM pictures of the areas are shown in Table 1 and are enlarged in Fig. 7.

A clear difference is evident in the relative orientation of the main lay of the surface with respect to the feed direction. While in zones $\mathrm{B}$ and $\mathrm{D}$, the feed and the lay of the surface are parallel, but this is not the case in zones A and $\mathrm{C}$. This is especially evident in the area machined with the $\mathrm{cBN}$ tool, and less evident when using a WC tool.

The surfaces machined with the $\mathrm{cBN}$ tool have a "foggy" appearance and a visually higher percentage of smeared material. Surfaces machined with the WC tool have a higher visual gloss and a limited presence of smeared material.

\section{Discussion}

\subsection{Surface Generation Mechanism: SEM Analysis}

The different tools and tool paths produce completely different surface topologies. As the light scattering is closely related to the orientation of the surface micro facets, the way in which the light is scattered is different as well. Taking a closer look at the generated surfaces while having in mind cutting direction and tool rotation, we can draw some conclusions about the way in which the surface is produced. Zones $\mathrm{A}$ and $\mathrm{C}$ have been cut when the tool was moving upward and downward, respectively, as described with respect to Fig. 3. The cutting speed results are always orthogonal to the feed direction, and the cut material is pushed and deformed over the yetto-be-machined surface in zone A, and over the freshlygenerated surface in zone $\mathrm{C}$. The tool marks in the feed direction are completely hidden by the smeared material generated by the teeth.

In zones $\mathrm{B}$ and $\mathrm{D}$, the situation is different: the cutting speed is parallel to the feed direction, and the material is pushed along that direction. The feed marks are clearly visible, and less smeared material is generated. These dynamics are emphasized in the areas machined by the cBN tool, which show a considerable amount of smeared material, as can be seen in Fig. 8. The presence of smeared material on the surface is not strictly related to $S a$ and $S q$; because they are amplitude parameters, the surface extension in the vertical direction is much more relevant. Most likely, the smeared material creates a pattern with low $z$ amplitude but higher slopes of the surface micro facets, thus influencing the average gradient of the surface rather than the average height.

\subsection{Amplitude Parameters: $S a$ and $S q$}

The lowest roughness values are reached in zone B, using the $\mathrm{cBN}$ tool. In zone $\mathrm{D}$, similar roughness values are measured for $\mathrm{cBN}$ and $\mathrm{WC}$ tools, while in the other areas, the differences are more pronounced. If a comparison with Table $\mathbf{1}$ is done, no close visual match with the amplitude roughness parameters and surface appearance can be identified; on the contrary, some areas with foggy visual appearance (surface $\mathrm{C}$ machined with $\mathrm{cBN}$, for example) show lower values of roughness than shiny surfaces (e.g., surface $\mathrm{C}$ machined with WC). 
Table 1. Comparison of SEM pictures of the different machined areas. The white arrow represents the feed direction.

\section{Surface topology of the different area of the test parts}

\section{Zone A: feed direction bottom to top of the pictures}

\begin{tabular}{|c|c|c|c|}
\hline \multirow{3}{*}{$\begin{array}{lll}\text { cBN zone } \mathbf{A} \\
\end{array}$} & WC zone A & \multirow{2}{*}{$\begin{array}{l}\text { Description cBN zone A } \\
\text { Significant smearing pattern } \\
\text { with main direction orthogonal } \\
\text { to the feed direction. Foggy } \\
\text { visual appearance. }\end{array}$} & \multirow{2}{*}{$\begin{array}{l}\text { Description WC zone A } \\
\text { Smooth visual appearance, } \\
\text { Smearing pattern orthogonal to } \\
\text { the feed direction, but almost } \\
\text { negligible. }\end{array}$} \\
\hline & + & & \\
\hline & $\frac{28 \times m}{28 \pi n}$ & $\begin{array}{ccc}S q \\
{[\mathrm{~nm}]}\end{array} \quad S d q \quad R d q$ & $\begin{array}{c}S q \\
{[\mathrm{~nm}]}\end{array}$ \\
\hline & & $\begin{array}{lll}0.199 & 0.194 & 39.2\end{array}$ & $0.036 \quad 0.033$ \\
\hline
\end{tabular}

\section{Zone B: feed direction left to the right of the pictures}

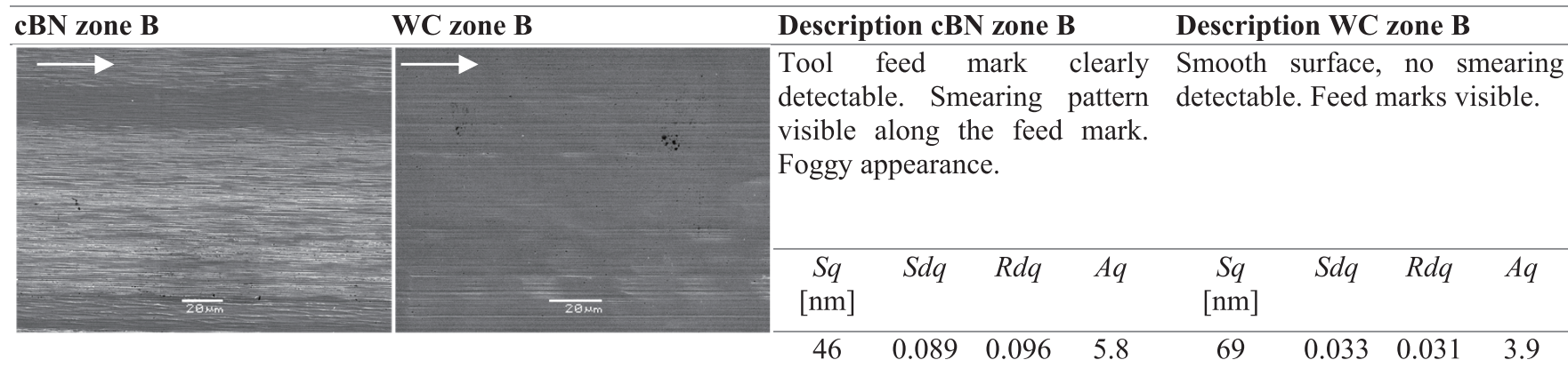

\section{Zone C: feed direction top to bottom of the pictures}

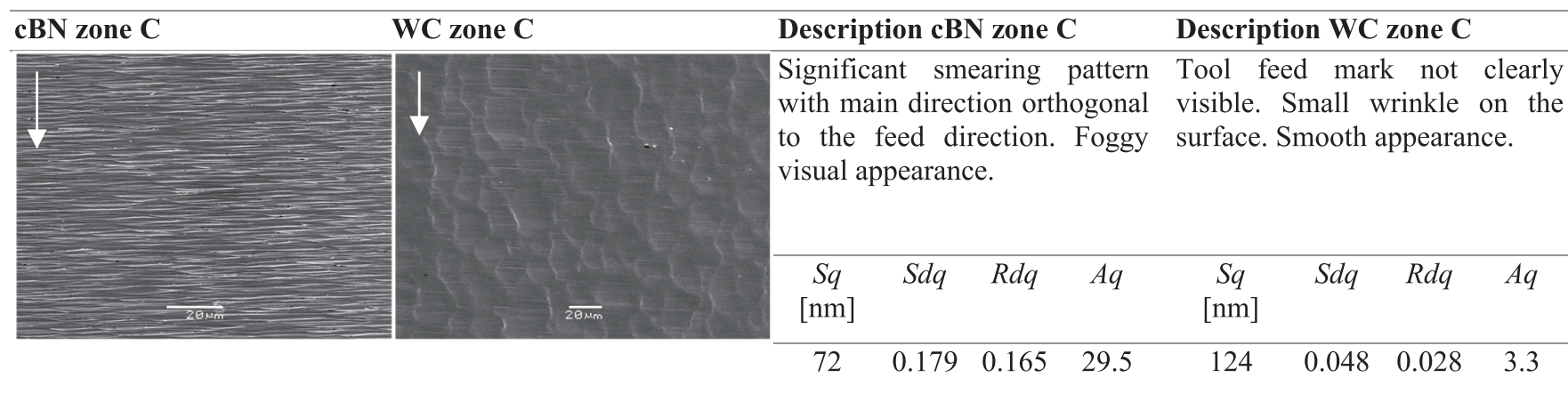

Zone D: feed direction right to left of the pictures

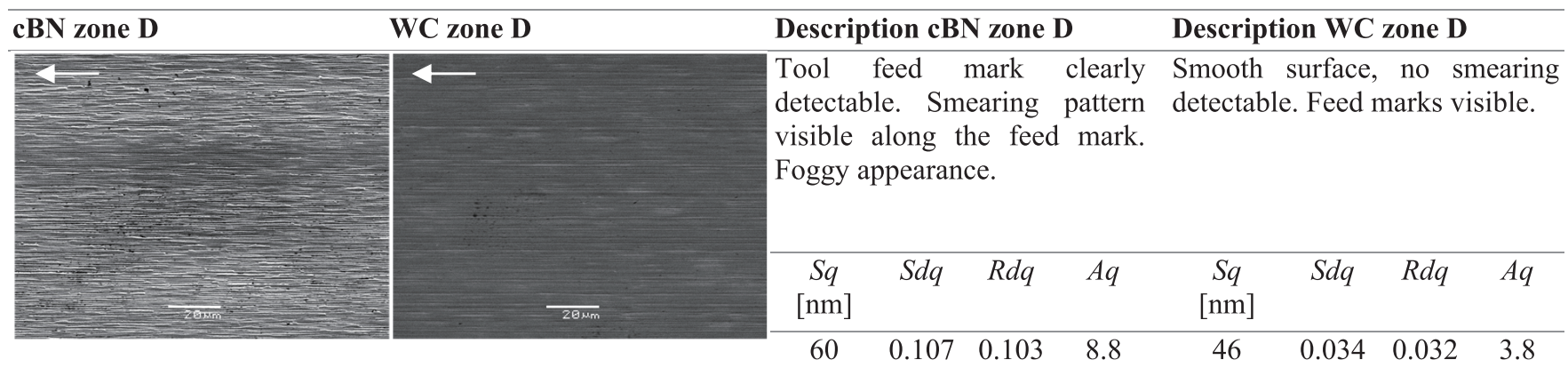




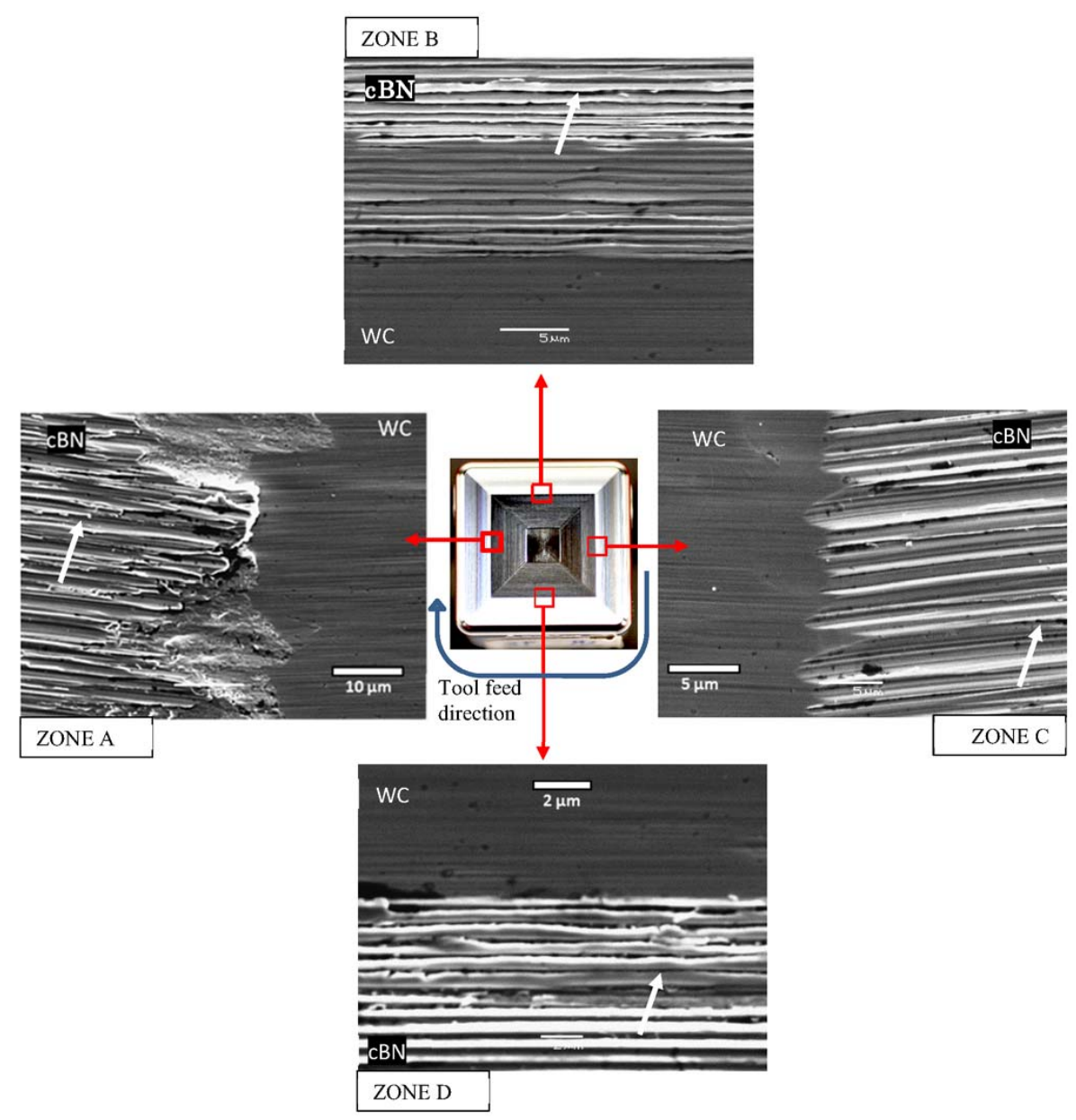

Fig. 7. The separation zone between the area machine with $\mathrm{cBN}$ and $\mathrm{WC}$ is shown in the SEM picture above for areas A, B, C, and D. The presence of smearing is evident in all the areas machined by $\mathrm{cBN}$. Some of the features produced by the smeared material are indicated with white arrows. In the center of the figure, a picture of the test sample is also shown. The locations of the SEM pictures over the surface are highlighted.
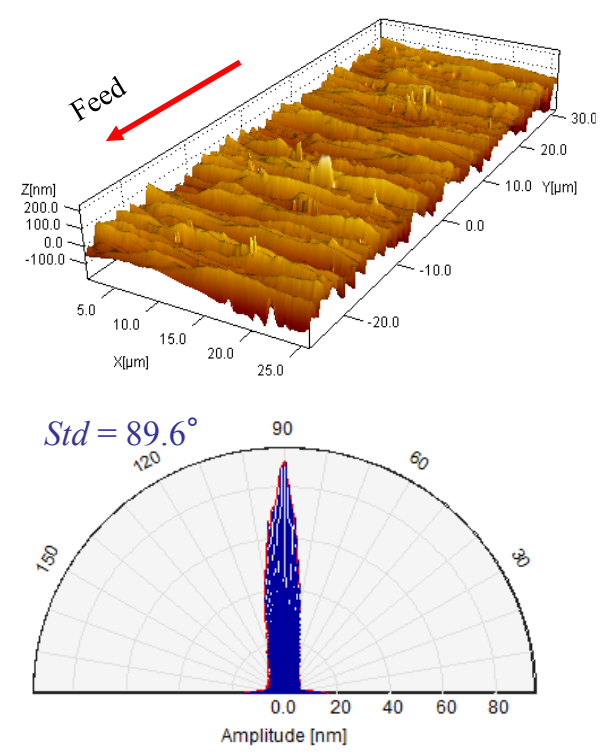

Fig. 8. Std calculations for surface $\mathrm{C}$ machined with a cBN tool.

\subsection{Surfaces Slopes Evaluation: $\boldsymbol{S d q}$}

The measured $S d q$ values are represented in Fig. 5 for the four different surfaces. $S d q$ is consistently lower in the area machined with WC tool, with a close match with the visual appearance of the SEM images of the surfaces. $S d q$ is a measurement of the average gradient of the surface slopes. Surface gradients, and thus surface angles, are mainly responsible for the distribution of the scattered light angle. This may explain the good visual correlation.

\subsection{Surfaces Slopes Evaluation: $\boldsymbol{R} \boldsymbol{d} \boldsymbol{q}$}

Before calculating $R d q$, some considerations need to be decided. $R d q$ is a 2-D parameter and therefore, the direction in which it is calculated has to be decided. If $R d q$ is compared with scattered light measurements, it should be calculated in the direction in which the light is scattered the most; thus, it should be calculated across the surface main lay. Examining the cutting dynamics, higher average slopes of the micro facets are expected along the cross-feed direction, while a smoother surface is generated along the feed which defines the main lay direction. 
Table 1 shows that this is not always true: in zones A and $\mathrm{C}$, the feed marks are completely covered by smeared material.

In order to detect the dominant lay direction, the Std parameter is calculated, as shown in Fig. 8.

Std is a roughness parameter representative of the direction of the dominant lay of the surface; the more the lay is parallel to the $x$-direction (in Fig. 8), the closer Std is to $90^{\circ}$. This roughness parameter is calculated for every area and once the dominant lay direction is known, $R d q$ is computed accordingly.

According to Std, the correct $R d q$ value to be considered is orthogonal to the feed direction in zones $\mathrm{B}, \mathrm{D}$, and $\mathrm{C}$ (machined with WC), and parallel in the others. As noticed for $S d q$, a strong correlation is identified with the visual appearance of the SEM images of Table 1.

\subsection{Light Scattering Measurement Evaluation: $A q$}

The intensity of the scattered light distribution is detected using a linear CCD array. Therefore, the sensor needs to be oriented consequently with the measuring direction. When a light beam strikes the surface, it is scattered in an elliptical shape with a main axis orthogonal to the surface main lay (as stated in the Optosurf Operating Manual). By orienting the sensor accordingly, it is possible to capture a larger part of the light distribution, obtaining a more significant value of $A q$.

Also, in the case of $A q$, the significant values are those measured orthogonal to the feed direction in zones B, D, and $\mathrm{C}$ (machined with WC), and parallel in the others.

The calculated $A q$ values are shown in Fig. 6. They follow the same trend recognized for $R d q$ and $S d q$, even though the variations among the different surfaces A, B, $\mathrm{C}$, and $\mathrm{D}$ are not the same.

\subsection{Comparison of $A q$ with $R d q$ and $S d q$}

When considering surface gloss and light scattering, the orientation of the surface micro facets plays a key role. It is reasonable to look for a relationship between parameters linked with the surface slopes ( $S d q$ and $R d q$ ) and with the scattered light distribution $(A q)$.

As observed before, all the three parameters are in close agreement with the visual appearance of the surfaces from the SEM images. However, before directly comparing them, the NA of the different instruments used for the measurements must be considered.

The scatterometer has a spot size of $900 \mu \mathrm{m}$ and a NA of 0.28 , meaning that most of the surface slopes with angles bigger than $\pm 8^{\circ}$ (half of the reflection angle) will scatter the light outside the detector, and thus will not contribute in the measurement of $A q$. On the other hand, the confocal microscope scans a square area of $250 \times 250 \mu \mathrm{m}$ with a NA of 0.95 , and can detect slopes up to $85^{\circ}$. When $R d q$ and $S d q$ are calculated from the confocal measurement, the value is computed while considering micro facets with higher angles than the maximum detectable angle by the detector array of the Optosurf. This may re-

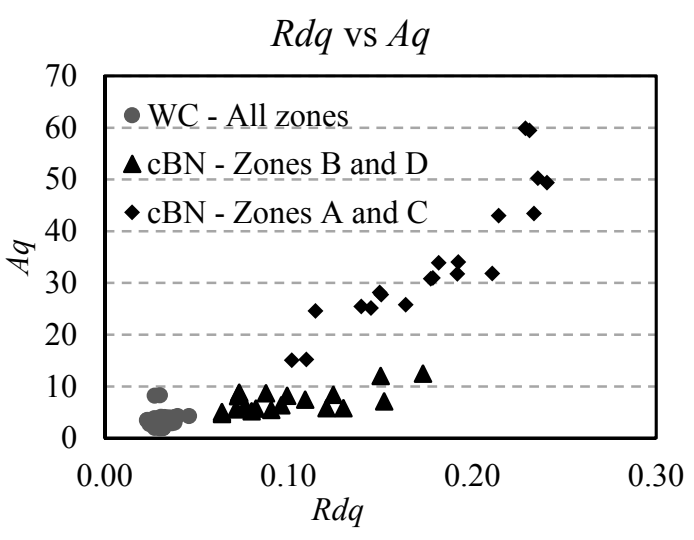

Fig. 9. $R d q$ and $A q$ values for all the experimental points. The three different trends of the data points are highlighted with different shapes.

sult in a shift of the experimental points toward higher values of $R d q(S d q)$.

\section{7. $R d q$ and Light Scattering Parameter $A q$}

For every data point of the different zones, $R d q$ and $A q$, as calculated orthogonal to the main lay direction, have been plotted. The data are shown in Fig. 9. The chart highlights three different trends in accordance with the tool feed direction.

The surfaces machined with the WC tool present a lower spread of the data points, indicating quite uniform surface quality, independent of the tool feed direction.

The points belonging to zones $\mathrm{A}$ and $\mathrm{C}$ machined with the $\mathrm{cBN}$ tool instead show a linear increase of $A q$ with the increase of the value of $R d q$, with an average $A q$ significantly higher than in areas B and D machined with the same tool. On the other hand, zones D and B show constant $A q$ values, independently of the variation of $R d q$.

Figure 9 also shows that even though comparable values of $R d q$ are measured for the surfaces machined with the cBN tool, a different behavior in term of light scattering is detected by the scattered light sensor.

\section{8. $S d q$ and Light Scattering Parameter $A q$}

$S d q$ is an extension in two dimensions of $R d q$, and hence a similar relation with $A q$ is expected.

Three different trends can also be noticed in this case, as shown in Fig. 10. They are not so pronounced as in the case of $R d q-A q$, but this can be ascribed to the unidimensional size of the CCD detector in the scatterometer with which $A q$ is calculated.

\subsection{Surface Slope Trends and Smeared Material}

The reasons why different trends occurred between $A q / S d q$ and $A q / R d q$ are still unclear. However, this phenomenon is emphasized in the areas machined with a cBN tool, in which the presence of smeared material is more pronounced (zones $\mathrm{A}$ and $\mathrm{C}$ ). 


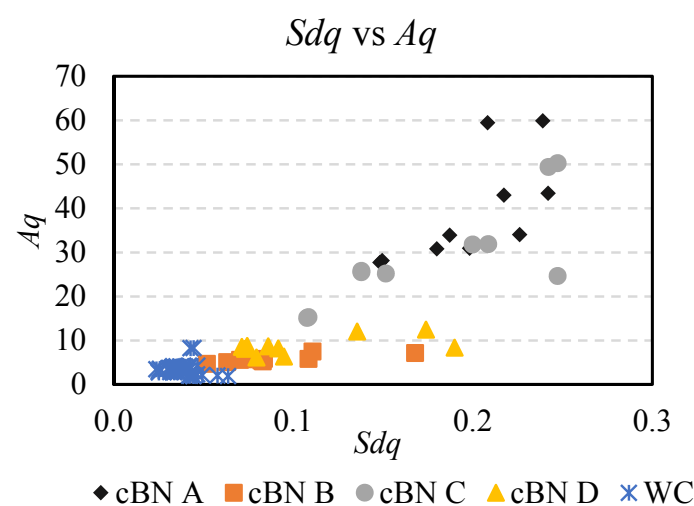

Fig. 10. $S d q$ and $A q$ values for all the experimental points. The areas machined by WC are grouped together as shown in the legend.

These discrepancies in the smearing patterns suggest that the topography of the smeared material can be the responsible for the different trends in the light scattering behavior.

$R d q$ and $S d q$ are a measurement of the average RMS slope of the surface, but they do not provide any information about the slope's spread and distribution. The high gradient features produced by smeared material are detected by the confocal microscope, and they give a great contribution to enhancing $R d q$ value. Due to the steep slopes, the smeared material can scatter the light at high angles and even out of the scattered light sensor range, and is therefore not contributing to the calculated $A q$ value.

\section{Conclusion}

In this work, a commercial scatterometer has been integrated in a milling machine to test the feasibility of inline measurements of milled parts. The results have been compared with confocal measurements of the same parts in a laboratory environment. The scattered light measurements provide a close match with the visual appearance of the part surfaces. The confocal measurements of $R d q$ and $S d q$ are in agreement with the overall trend of the gloss of the surface if the surfaces present similar topology. Two different trends in the relation between the roughness parameters $R d q$ and $S d q$ have been detected. The areas machined with a cBN tool show different ratios of $A q / R d q$ depending on the tool feed direction. The smeared material on the part is assumed to be the responsible for the significant differences in the $A q$ value. In zones $\mathrm{A}$ and $\mathrm{C}$, the more emphasized smearing pattern leads to higher $A q$ values compared with zones B and D. The well-established roughness parameters $S a$ and $S q$ failed in describing the appearance of the surface. As amplitude parameters, their correlation with the surface gloss is significant only to a certain extent.

\section{References:}

[1] K. D. Thoben, S. Wiesner, and T. Wuest, “'Industrie 4.0' and Smart Manufacturing - A Review of Research Issues and Application Examples," Int. J. Automation Technol., Vol.11, No.1, pp. 4-19, 2017.

[2] Y. Kobayashi, K. Shirai, Y. Hara, T. Mizoguchi, and K. Kawasaki, "Generation and Assessment of Random Surface Texture over a Wide Area," Int. J. Automation Technol., Vol.5, No.2, pp. 185-189, 2011.

[3] T. Hirose, Y. Kami, and T. Shimizu, "Development of On-Machine Measurement Unit for Correction Processing of Aspheric Lens Mold with High Numerical Aperture," Int. J. Automation Technol., Vol.8, No.1, pp. 34-42, 2014.

[4] A. Schöch, A. Salvadori, I. Germann, S. Balemi, C. Bach, A. Ghiotti, S. Carmignato, A. L. Maurizio, and E. Savio, "Highspeed measurement of complex shaped parts at elevated temperature by laser triangulation," Int. J. Automation Technol., Vol.9, No.5, pp. 558-566, 2015.

[5] I. S. Jawahir, E. Brinksmeier, R. M'Saoubi, D. K. Aspinwall, J. C. Outeiro, D. Meyer, D. Umbrello, and A. D. Jayal, "Surface integrity in material removal processes: Recent advances," CIRP Ann. - Manuf. Technol., Vol.60, No.2, pp. 603-626, 2011.

[6] M. Shimizu, H. Sawano, H. Yoshioka, and H. Shinno, "On-Machine Surface Texture Measuring System Using Laser Speckle Pattern Analysis," Int. J. Automation Technol., Vol.10, No.1, pp. 69-77, 2016.

[7] L. De Chiffre, P. Lonardo, H. Trumpold, D. A. Lucca, G. Goch, C. A. Brown, J. Raja, and H. N. Hansen, "Quantitative Characterisation of Surface Texture," CIRP Ann. - Manuf. Technol., Vol.49, No.2, pp. 635-652, 2000.

[8] L. De Chiffre, H. Kunzmann, G. N. Peggs, and D. A. Lucca, "Surfaces in precision engineering, microengineering and nanotechnology," CIRP Ann. - Manuf. Technol., Vol.52, No.2, pp. 561-577, 2003.

[9] T. Hayashi, Y. Takaya, N. Motoishi, and Y. Nakatsuka, "Surface Inspection of Micro Glass Lens Mold Based on Total Angle Resolved Scattering Characterization," Int. J. Automation Technol., Vol.4, No.5, pp. 1-7, 2010.

[10] H. N. Hansen, K. Carneiro, H. Haitjema, and L. De Chiffre, "Dimensional micro and nano metrology," CIRP Ann. - Manuf. Technol., Vol.55, No.2, pp. 721-743, 2006 .

[11] A. R. Hanson, "Measurement Good Practice Guide No.94, Good Practice Guide for the Measurement of Gloss," National Physical Laboratory, 2006.

[12] ASTM D523-14, "Standard Test Method for Specular Gloss," ASTM Int., 2018.

[13] J. Seewig, G. Beichert, R. Brodmann, H. Bodschwinna, and M. Wendel, "Extraction of shape and roughness using scattering light," Proc. SPIE, Vol.7389, p. 73890N-1-11, 2009.

[14] L. Pilný and G. Bissacco, "Development of on the machine process monitoring and control strategy in Robot Assisted Polishing," CIRP Ann. - Manuf. Technol., Vol.64, pp. 313-316, 2015.

[15] T. Nomura, T. Nagasaki, and M. Ito, "Development of Inspection Machine that Detect Small Particles Added on Surface with Precise Pattern by Capturing Backwards Scattered Polarized Light," Int. J. Automation Technol., Vol.6, No.6, pp. 781-791, 2012.

[16] L. Pilný, G. Bissacco, and L. De Chiffre, "Validation of in-line surface characterization by light scattering in Robot Assisted Polishing," Proc. of the 3rd Int. Conf. on Virtual Machining Process technology (VMPT), p. 8, 2014.

[17] Verband der Automobilindustrie, "VDA 2009, Geometrische Produktspezifikation Oberflächenbeschaffenheit Winkelaufgelöste Streulichtmesstech-Nik Definition, Kenngrøßen Und Anwendung (Light Scattering Measurement Tech.)," 2009.

[18] R. Bordmann and J. Seewig, "Non-Contact Surface Metrology by Means of Light Scattering," Q. J. Wang and Y.-W. Chung (Eds.) "Encyclopedia of Tribology," Springer, pp. 2434-2440, 2013.

[19] J. Bohm, M. Jech, and M. Vellekoop, "Analysis of NM-Scale Scratches on High-Gloss Tribological Surfaces by Using an AngleResolved Light Scattering Method," Tribol. Lett., Vol.37, No.2, pp. 209-214, 2010.

[20] N. A. Feidenhans'l, P. Hansen, L. Pilný, M. H. Madsen, G. Bissacco, J. C. Petersen, and R. Taboryski, "Comparison of optical methods for surface roughness characterization," Meas. Sci. Technol., Vol.26, No.8, p. 085208, 2015. 


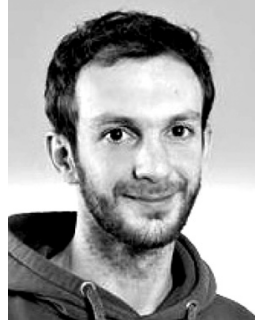

Name:

Francesco Giuseppe Biondani

\section{Affiliation:}

Technical University of Denmark

Address:

Produktionstorvet, Kgs. Lyngby 2800, Denmark

Brief Biographical History:

2014-2017 Ph.D. at Technical University of Denmark

2017- Postdoc, Technical University of Denmark

Main Works:

- Ph.D. thesis: Process chains for advance tooling based on additive manufacturing

- F. G. Biondani, G. Bissacco, P. T. Tang, and H. N. Hansen, "Additive Manufacturing of Mould Inserts with Mirror-like Surfaces," Procedia CIRP, Vol.68, pp. 369-374, 2018.

Membership in Academic Societies:

- European Society for Precision Engineering and Nanotechnology (euspen)

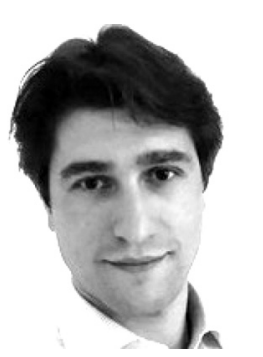

Name:

Giuliano Bissacco

\section{Affiliation:}

Department of Mechanical Engineering, Technical University of Denmark

\section{Address:}

Produktionstorvet, Kgs. Lyngby 2800, Denmark

Brief Biographical History:

2001 Received Ph.D. (Micro Manufacturing) from Technical University of Denmark

2004- Postdoc, Technical University of Denmark

2005- Assistant Professor, Technical University of Denmark

2008- Assistant Professor / Research Unit Manager, University of Padova

2010- Associate Professor, Technical University of Denmark

Main Works:

- L. Pilný and G. Bissacco, "Development of on the machine process monitoring and control strategy in Robot Assisted Polishing," CIRP

Annals - Manufacturing Technology, Vol.64, pp. 313-316, 2015.

- G. Bissacco, G. Tristo, H. N. Hansen, and J. Valentincic, "Reliability of electrode wear compensation based on material removal per discharge in micro EDM milling," Annals of the CIRP, Vol.62, No.1, pp. 179-182, 2013.

- G. Bissacco, H. N. Hansen, and J. Slunsky, "Modelling the Cutting Edge Radius Size Effect for Force Prediction in Micro Milling," Annals of the CIRP, Vol.57, No.1, pp. 113-116, 2008.

- L. Alting, F. Kimura, H. N. Hansen, and G. Bissacco, "Micro Engineering," CIRP Annals, Vol.52, No.2, pp. 635-658, 2003

\section{Membership in Academic Societies}

- International Academy for Production Engineering (CIRP)

- European Association on Multi Material Micro Manufacturing (4M)

- Danish Academy of Technical Sciences (ATV-Semapp), Steering

Committee of the Group for Production and Technological Innovation
Name:

Lukáś Pilný

\section{Affiliation:}

Senior Device Engineer, Novo Nordisk A/S

Address:

Brennum Park 24k, Hillerød 3400, Denmark

Brief Biographical History:

2015- Postdoc, Technical University of Denmark

2016- Senior Device Engineer, Technical University of Denmark

Main Works:

- L. Pilný and G. Bissacco, "Development of on the machine process monitoring and control strategy in Robot Assisted Polishing," CIRP Ann. Manuf. Technol., Vol.64, pp. 313-316, 2015.

- L. Pilný, G. Bissacco, L. De Chiffre, and J. Ramsing, "Acoustic

Emission Based In-process Monitoring of Surface Generation in Robot

Assisted Polishing," Int. J. of Computer Integrated Manufacturing, Vol.29, No.11, pp. 1218-1226, 2016.

- N. A. Feidenhans'l, P. Hansen, L. Pilný, M. H. Madsen, G. Bissacco, J. C. Petersen, and R. Taboryski, "Comparison of optical methods for surface roughness characterization,” Meas. Sci. Technol., Vol.26, No.8, p. 085208 , 2015.

Membership in Academic Societies:

- European Society for Precision Engineering and Nanotechnology (euspen)

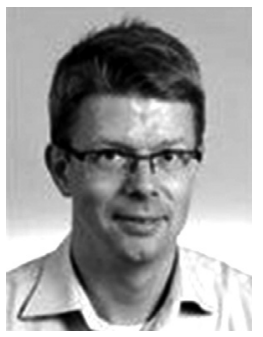

Name:

Hans Nørgaard Hansen

Affiliation:

Professor, Technical University of Denmark

Address:

Produktionstorvet, Kgs. Lyngby 2800, Denmark

Brief Biographical History:

1997 Received Ph.D. from Technical University of Denmark

2002- Professor, Technical University of Denmark

2016- Head of Department of Mechanical Engineering, Technical

University of Denmark

Main Works:

- metrology, micro/nano manufacturing

Membership in Academic Societies:

- European Society of Precision Engineering (euspen)

- International Academy for Production Engineering (CIRP) 